\title{
La maladie de Parkinson due aux mutations de la parkine
}

$\mathbf{L}$ a maladie de Parkinson est une affection neurodégénérative fréquente dont la prévalence atteint $2 \%$ après l'âge de 70 ans. Elle se manifeste cliniquement par la triade akinésie, rigidité et tremblement de repos, qui répond favorablement, au moins au début de l'évolution, au traitement substitutif par la lévodopa. Ces troubles sont dus à une perte neuronale qui affecte préférentiellement les neurones dopaminergiques de la voie nigro-striatale. Les lésions sont accompagnées d'un marqueur histopathologique, le corps de Lewy, inclusion neuronale cytoplasmique éosinophile et ubiquitinylée. Les formes monogéniques sont considérées comme rares mais déjà plusieurs gènes responsables de maladie de Parkinson ont été récemment identifiés: l' $\alpha$-synucléine $\left(\mathrm{m} / \mathrm{s} 2000, n^{\circ} 8\right.$-9, p. 956) et l'ubiquitine carboxy terminal hydrolase L1 (UCH-L1) dans les formes autosomiques dominantes, et la parkine dans les formes autosomiques récessives.

\section{Les formes autosomiques} dominantes de maladie de Parkinson

Les deux gènes impliqués dans les atteintes autosomiques dominantes correspondent à des formes très rares. Deux mutations de l' $\alpha$-synucléine ont été décrites, l'une dans un petit nombre de familles italo-américaine et grecques qui proviennent d'un effet fondateur $([1,2]$ et $\mathrm{m} / \mathrm{s}$ 1997, $n^{\circ} 10, p .1218$ ) et l'autre dans une famille allemande [3]. Deux membres d'une famille allemande portent une mutation du gène UCHL1 qui réduit de $50 \%$ environ son activité enzymatique [4]. Bien que les mutations de ces gènes soient très rarement impliquées dans les formes familiales de maladie de Parkinson,

études génétiques, leur identification pourrait permettre de comprendre certaines étapes du processus pathologique. Il est intéressant de constater que l' $\alpha$-synucléine constitue un composant majeur des corps de Lewy présents dans la maladie de Parkinson idiopathique ou dans la démence avec corps de Lewy. De plus, la surexpression de l' $\alpha$-synucléine chez la souris et la drosophile s'est révélée toxique pour les neurones, particulièrement les neurones dopaminergiques $\left([5,6]\right.$ et $m / s$ 2000, $n^{\circ} 8$-9, p. 956). Les souris transgéniques pour l' $\alpha$-synucléine humaine ne présentent pas de véritables corps de Lewy mais une accumulation d' $\alpha$ synucléine dans les neurites qui pourraient correspondre au neuropil threads décrits dans la maladie humaine [5]. En revanche, chez la drosophile, les agrégats sont intracytoplasmiques comme dans les corps de Lewy, voire même intranucléaires dans certains cas. De plus, en microscopie électronique, des fibrilles de 7 à $10 \mathrm{~nm}$ sont observées au pourtour des agrégats [6].

Les formes autosomiques récessives et le gène de la parkine

Les formes autosomiques récessives ont été individualisées au Japon sous le terme de parkinsonisme juvénile autosomique récessif (autosomal recessive juvenile parkinsonism - ARJP) ([7, 8] et $m / s$ 1998, $\left.n^{\circ} 8-9, p .971\right)$. Dans les familles japonaises, le tableau clinique se caractérise par un début précoce, souvent avant l'âge de 20 ans, une dystonie des membres inférieurs, des réflexes vifs, une amélioration nette par le sommeil, ainsi qu'une excellente réactivité à la Levodopa associée à la survenue précoce de dyskinésies. Lorsque le gène fut localisé sur le bras long du chro- mosome 6 dans les familles japonaises [9], il fut possible de montrer par des études de liaison génétique l'existence d'une même entité aux Etats-Unis, en Europe et en Afrique du Nord $\left([10,11]\right.$ et $\mathrm{m} / \mathrm{s} 1998, n^{\circ} 12$, p. 1451). L'année suivante, Kitada et $a l$. identifièrent le gène responsable qu'ils dénommèrent Parkine [12]. Il s'agit d'un gène nouveau, qui comporte douze exons codants et s'étend sur plus de $500 \mathrm{~kb}$. Il code pour une protéine de fonction inconnue qui comporte 465 acides aminés.

\section{La grande diversité des mutations de la parkine}

Les premières mutations décrites étaient des délétions homozygotes d'exons, rapidement complétées par la caractérisation de nombreuses mutations ponctuelles [12-20] (figure 1). L'étude la plus complète fut menée dans le cadre du réseau européen dédié à l'étude de la génétique de la maladie de Parkinson [20]. Son intérêt est double, d'une part, elle rassemble un grand nombre de familles $(n=73)$ et de cas isolés $(n=100)$ d'origine principalement européenne, et d'autre part, elle combine différentes approches méthodologiques pour caractériser la grande variété des mutations qui affectent le gène de la parkine. En effet, l'observation de délétions de différents exons à l'état homozygote permettait de suspecter l'existence de délétions à l'état hétérozygote dans des familles non consanguines. Ce type de réarrangement n'est pas détecté par le séquençage du gène. C'est pourquoi une technique de PCR «multiplexe» semi-quantitative fut développée pour explorer l'ensemble de la séquence codante du gène de la parkine, à l'exception de l'exon 1. Ainsi, 19 réarrangements 


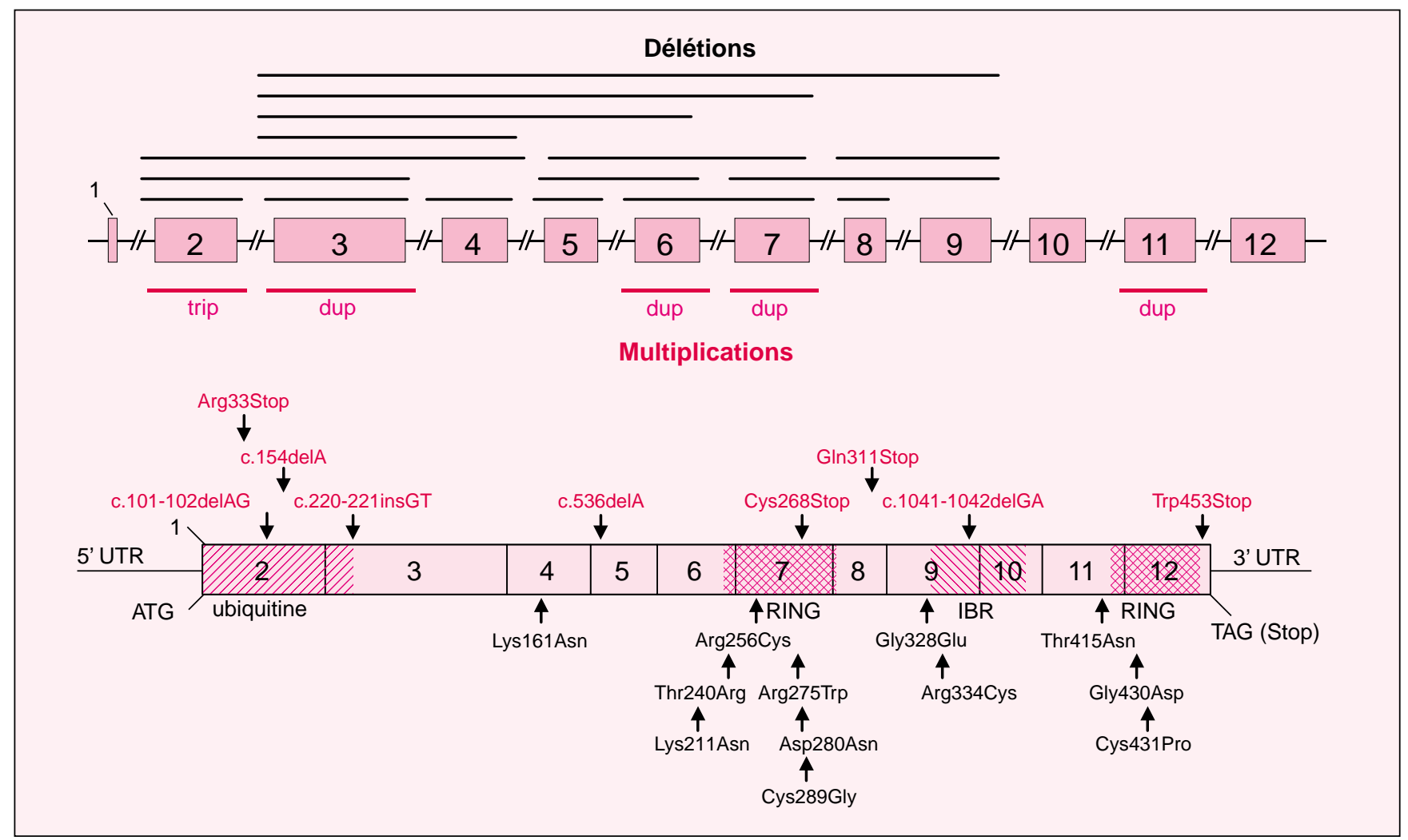

Figure 1. Mutations du gène de la parkine et maladie de Parkinson [12-20]. A. Le gène de la parkine comprend douze exons. Les différents réarrangements décrits sont soit des délétions (en noir), soit des multiplications (en rouge). Les traits indiquent la taille des réarrangement et les exons impliqués. Trip: triplication; dup: duplication. B. Mutations ponctuelles. Les mutations produisant des protéines tronquées sont indiquées en rouge et les mutations faux-sens en noir. Pour les mutations qui créent un décalage du cadre de lecture, les nucléotides délétés ou insérés sont indiqués ainsi que leur position dans l'ADN complémentaire. Les domaines "ubiquitine" et "RING-IBR-RING" sont hachurés.

différents du gène de la parkine furent caractérisés (figure 1A). Il s'agit de 14 combinaisons variables de délétions d'exons et de 5 multiplications d'exons [20]. De façon surprenante, non seulement des duplications mais aussi une triplication d'exons furent détectées. L'existence de délétions et de duplications des mêmes exons permet de suspecter qu'un mécanisme de recombinaison inégale soit à l'origine de ces réarrangements. Cette hypothèse pourra être confirmée si l'analyse des points de cassure révèle qu'ils sont identiques pour les délétions et duplications des mêmes exons. Les mutations ponctuelles sont également très fréquentes (figure 1B). Certaines produisent une protéine tronquée, mais la majorité est de type faux-sens. La plus grande partie de ces dernières est située dans la moitié C-terminale de la protéine. $\mathrm{Vu}$ le mode de transmission, les deux types de mutations sont susceptibles d'entraîner une perte de fonction de la protéine.

\section{Fréquence des mutations de la parkine}

Globalement, les mutations de la parkine sont trouvées dans près de $50 \%$ des formes familiales autosomiques récessives de début précoce (avant l'âge de 45 ans) [20]. Elles sont également retrouvées dans des cas isolés de début précoce avec une fréquence moindre $(18 \%)$. Néanmoins, la proportion de cas isolés liée à une altération du gène de la Parkine décroît rapidement lorsque l'âge de début augmente. Elle atteint $77 \%$ lorsque la maladie débute avant l'âge de 20 ans mais tombe à $25 \%$ environ pour un début entre 21 et 30 ans et ne dépasse guère quelques pourcents pour un début après l'âge de 30 ans [20]. Il est probable que les cas isolés dus à des mutations du gène de la Parkine sont progressivement dilués par des cas de maladie de Parkinson idiopathique dont la proportion augmente avec l'âge.

\section{Phénotype correspondant aux mutations de la parkine}

L'évaluation d'une grande série de patients a permis de mieux définir le phénotype correspondant aux mutations de ce gène [20]. La première surprise fut l'observation d'âges de début très variables, entre 7 et 58 ans dans cette série, qui démontre qu'il ne s'agit pas toujours d'un syndrome parkinsonien de début juvénile (avant 20 ans) ou même précoce (avant 40 ans). La comparaison entre les 
formes à début précoce dues à des mutations de la parkine et les formes précoces sans mutation ne met en évidence aucun signe spécifique d'un groupe de patients. Cependant, l'âge de début est significativement plus précoce dans le groupe parkine, de même que la fréquence d'une dystonie inaugurale ou de réflexes vifs à l'examen. De plus, les patients porteurs de mutations du gène de la parkine répondent mieux à la lévodopa, mais présentent plus fréquemment des dyskinésies induites par le traitement par rapport aux patients sans mutation. En revanche, la fréquence des autres signes cliniques, ainsi que la sévérité de la maladie sont similaires dans les deux groupes. Deux éléments importants distinguent les patients parkine de la maladie de parkinson idiopathique. Les patients parkine ont un âge de début moyen beaucoup plus précoce et une progression nettement plus lente de la maladie, contrairement à de nombreuses maladies neurodégénératives pour lesquelles la sévérité augmente lorsque l'âge de début diminue. Ainsi, le temps mis pour atteindre chaque stade de l'échelle Hoehn et Yahr est environ quatre fois plus long chez les porteurs d'une mutation du gène de la parkine que dans la maladie de Parkinson idiopathique. Dans la pratique clinique, la mise en évidence d'une mutation du gène de la parkine constitue un facteur de bon pronostic puisqu'il s'agit souvent de formes peu évolutives, dont la réponse à la levodopa est excellente, et ne s'épuise pas. Cette note optimiste doit être tempérée par la grande fréquence des dyskinésies et des fluctuations induites par la levodopa, qui peuvent rendre l'équilibration du traitement difficile. Cependant, la stimulation cérébrale profonde, utilisée avec succès chez certains patients atteints de maladie de Parkinson idiopathique, s'est révélée efficace chez un patient parkine [21].

\section{La neuropathologie des cas parkine}

Seuls trois cas neuropathologiques de patients porteurs de mutations de la parkine [22] ou liés au même locus [23] ont été décrits. Les sive des neurones dopaminergiques de la substantia nigra pars compacta, associée à une baisse de l'immunoréactivité pour la tyrosine hydroxylase, enzyme qui catalyse l'étape limitante de la synthèse des catécholamines. Cette perte peut être mise en évidence indirectement chez les patients par tomographie par émission de positrons qui montre une diminution marquée de la capture de fluorodopa dans le putamen et le noyau caudé, comparable à celle observée dans les formes avancées de maladie de Parkinson idiopathique [21]. Ces anomalies rendent bien compte du syndrome parkinsonien observé. Cependant, l'examen neuropathologique révèle aussi une perte neuronale modérée dans le locus coeruleus. La différence majeure avec la maladie de Parkinson idiopathique est l'absence de corps de Lewy dans la substantia nigra ou d'autres régions du système nerveux. En revanche, des astrocytes immunoréactifs pour la protéine tau sont visualisés, comme dans la paralysie supranucléaire progressive ou la maladie de Pick dont les bases moléculaires sont différentes.

\section{L'expression de la parkine dans le système nerveux}

Des anticorps dirigés contre la parkine ont été produits pour étudier la distribution cérébrale de la protéine [24, 25]. Un marquage, en majorité des neurones mais aussi de quelques cellules gliales, est observé dans la plupart des régions cérébrales. Dans les neurones, les corps cellulaires et les prolongements nerveux sont marqués mais pas les noyaux. L'intensité du marquage varie selon les structures mais est importante dans les noyaux gris centraux, particulièrement la substantia nigra et le globus pallidus. La distribution de l'immunomarquage est tout à fait superposable chez le rat, le marmoset, le singe et l'homme [25]. Chez les patients atteints de maladie de Parkinson, l'immunomarquage est diminué dans la substantia nigra en raison de la perte neuronale mais les corps de Lewy sont peu ou pas marqués par la parkine. Contrairement à l' $\alpha$-synucléine, la parkine ne semble donc pas constituer un composant important des corps de Lewy. Il existe une discordance entre la distribution ubiquitaire de la Parkine dans le système nerveux de différentes espèces et la grande sélectivité des lésions observées en l'absence de la protéine chez l'homme. Cette observation suggère que la Parkine pourrait jouer un rôle important dans le développement et/ou la survie des neurones dopaminergiques de la substantia nigra, mais pas d'autres régions, ou bien que d'autres protéines peuvent suppléer son absence dans les neurones qui ne sont pas affectés.

\section{Quelle est la fonction de la parkine?}

L'analyse de la séquence de la parkine révèle la présence de deux motifs. Le premier, à l'extrémité $\mathrm{N}$ terminale de la protéine, montre une homologie modérée avec l'ubiquitine, et le second, dans la moitié Cterminale, consiste en un domaine RING-IBR-RING (IBR : in-betweenring) [26]. L'importance de ce dernier motif dans la parkine est soulignée par le fait qu'il est très conservé entre les espèces et qu'il constitue la cible préférentielle des mutations ponctuelles de type faux-sens. Il est à noter que ces mutations touchent probablement des acides aminés fonctionnellement importants, puisqu'elles produisent un phénotype indistinguable des mutations tronquantes responsables d'une perte de fonction complète [20]. Les domaines RING-IBR-RING ont été identifiés dans de nombreuses protéines, dont il a été récemment montré qu'elles interviennent comme ubiquitine ligase E3 dans le processus d'ubiquitinylation [27-30]. Ces protéines déterminent en partie la spécificité de reconnaissance des protéines qui seront ubiquitinylées. Tout récemment, il a été démontré que la parkine fonctionne comme une ubiquitine ligase E3, qui interagit avec des enzymes de conjugaison à l'ubiquitine $\mathrm{E} 2$ ( $\mathrm{UbcH} 7$ et $\mathrm{UbcH} 8$ ) via le domaine RING-IBR-RING et avec des substrats putatifs d'ubiquitinylation via le domaine homologue à l'ubiquitine [31]. Il est donc facile de spéculer sur l'absence de corps de Lewy, structure fortement ubiquitinylée, 
chez les porteurs de mutations du gène de la parkine. En revanche, les protéines cibles (substrats d'ubiquitinylation), qui pourraient expliquer la sélectivité de la mort neuronale, ne sont pas encore connues. Il reste donc beaucoup à faire pour déterminer les mécanismes de la mort des neurones dopaminergiques due à l'absence de parkine. L'inactivation du gène de la parkine chez la souris ou dans des lignées neuronales devrait considérablement aider à préciser sa fonction et les conséquences de son absence

\section{RÉFÉRENCES}

1. Polymeropoulos MH, Lavedan C, LeroyE, et al. Mutation in the alpha-synuclein gene identified in families with Parkinson's disease. Science 1997; 276 : 2045-7.

2. Athanassiadou A, Voutsinas G, Psiouri L et al. Genetic analysis of families with Parkinson disease that carry the Ala53Thr mutation in the gene encoding alpha-synuclein. Am J Hum Genet 1999; 65: 555-8.

3. Krüger R, Kuhn W, Müller T, et al. Ala30Pro mutation in the gene encoding alpha-synuclein in Parkinson's disease. Nat Genet 1998; 18 : 106-8.

4. Leroy E, Boyer R, Auburger G, et al. The ubiquitin pathway in Parkinson's disease. Nature 1998; 395: 451-2.

5. Masliah E, Rockenstein E, Veinbergs I, et al. Dopaminergic loss and inclusion body formation in alpha-synuclein mice : implications for neurodegenerative disorders. Science 2000 ; 287 : 1265-9.

6. Feany MB, Bender WW. A Drosophila model of Parkinson's disease. Nature 2000 404: 394-8.

7. Yamamura Y, Sobue I, Ando K, Iida M, Yanagi T. Paralysis agitans of early onset with marked diurnal fluctuation of symptoms. Neurology $1973 ; 23$ : 239-44.

8. Ishikawa A, Tsuji S. Clinical analysis of 17 patients in 12 Japanese families with autosomal- recessive type juvenile parkinsonism. Neurology 1996 ; 47 : 160-6.

9. Matsumine H, Saito M, Shimoda Matsubayashi S, et al. Localization of a gene for an autosomal recessive form of juvenile Parkinsonism to chromosome 6q25.2-27. Am J Hum Genet 1997; 60 : 588-96.

10. Tassin J, Dürr A, de Broucker T, et al. Chromosome 6-linked autosomal recessive early-onset parkinsonism: linkage in european and algerian families, extension of the clinical spectrum, and evidence of a smal homozygous deletion in one family. $\mathrm{Am} J$ Hum Genet 1998; 63: 88-94.

11. Jones AC, Yamamura Y, Almasy L, et al. Autosomal recessive juvenile parkinsonism maps to 6q25.2-q27 in four ethnic groups: detailed genetic mapping of the linked region. Am J Hum Genet 1998; 63: 80-7.

12. Kitada T, Asakawa S, Hattori N, et al. Mutations in the parkin gene cause autosomal recessive juvenile parkinsonism. Nature 1998; 392: 605-8

13. Hattori N, Matsumine $\mathrm{H}$, Asakawa S, et al. Point mutations (Thr240Arg and Gln311Stop) in the parkin gene. Biochem Biophys Res Commun 1998; 249: 754-8.

14. Hattori N, Kitada $\mathrm{T}$, Matsumine $\mathrm{H}$, et al. Molecular genetic analysis of a novel parkin gene in Japanese families with autosomal recessive juvenile parkinsonism: evidence for variable homozygous deletions in the parkin gene in affected individuals. Ann Neurol 1998; 44: 935-41.

15. Lücking $\mathrm{CB}$, Abbas N, Dürr A, et al. Homozygous deletions in parkin gene in European and North African families with autosomal recessive juvenile parkinsonism. Lancet 1998; 352 : 1355-6.

16. Leroy E, Anastasopoulos D, Konitsiotis S, Lavedan C, Polymeropoulos MH. Deletions in the parkin gene and genetic heterogeneity in a Greek family with early onset Parkinson's disease. Hum Genet 1998; 103 . 424-7.

17. Abbas N, Lücking CB, Ricard S, et al. A wide variety of mutations in the parkin gene are responsible for autosomal recessive parkinsonism in Europe. Hum Mol Genet 1999; 8: $567-74$.

18. Nisipeanu P, Inzelberg R, Blumen, et al. Autosomal-recessive juvenile parkinsonism in a Jewish Yemenite kindred: mutation of Parkin gene. Neurology 1999; 53: 1602-4.

19. Maruyama M, Ikeuchi T, Saito M, et al. Mutational analysis of the parkin gene and genotype-phenotype correlations in patients with autosomal recessive juvenile parkinsonism. Ann Neurol 2000; 48: 245-50.

20. Lücking CB, Dürr A, Bonifati V, et al. Association between early-onset Parkinson's disease and mutations in the parkin gene. $N$ Engl J Med 2000; 342: 1560-7.

21. Broussolle E, Lücking CB, Ginovart N Pollak P, Remy P, Dürr A. [ ${ }^{18}$ F]Dopa PET study in patients with young-onset Parkinson's disease and parkin gene mutations. Neurology 2000 (sous presse).

22. Mori H, Kondo T, Yokochi M, et al. Pathologic and biochemical studies of juvenile parkinsonism linked to chromosome 6q. Neurology 1998; 51 : 890-2.

23. Takahashi H, Ohama E, Suzuki S, et al. Familial juvenile parkinsonism: clinical and pathologic study in a family. Neurology 1994; 44: 437-41.

24. Shimura H, Hattori N, Kubo S, et al. Immunohistochemical and subcellular localization of Parkin protein: absence of protein in autosomal recessive juvenile parkinsonism patients. Ann Neurol 1999; 45: 668-72.
25. Gu WJ, Abbas N, Lagunes MZ, et al. Cloning of rat parkin CDNA and distribution of parkin in rat brain. J Neurochem $2000 ; 74$ : 1773-6.

26. Morett E, Bork P. A novel transactivation domain in parkin. Trends Biochem Sci $1999 ; 24: 229-31$.

27. Lorick KL, Jensen JP, Fang S, Ong AM, Hatakeyama S, Weissman AM. RING fingers mediate ubiquitin-conjugating enzyme (E2)-dependent ubiquitination. Proc Natl Acad Sci USA 1999; 96: 11364-9.

28. Joazeiro CA, Wing SS, Huang H, Leverson JD, Hunter T, Liu YC. The tyrosine kinase negative regulator c-Cbl as a RINGtype, E2-dependent ubiquitin-protein ligase. Science 1999; 286 : 309-12.

29. Yang Y, Fang S, Jensen JP, Weissman AM, Ashwell JD. Ubiquitin protein ligase activity of IAPs and their degradation in proteasomes in response to apoptotic stimuli. Science 2000 ; 288: 874-7.

30. Chen A, Wu K, Fuchs SY, Tan P, Gomez C, Pan ZQ. The conserved RING-H2 finger of ROC1 Is required for ubiquitin ligation. $J$ Biol Chem 2000; 275 : 15432-9.

31. Shimura H, Hattori N, Kubo S, et al. Familial Parkinson disease gene product, parkin, is a ubiquitin-protein ligase. Nat Genet 2000; 25: 302-5.

Ce travail a bénéficié du soutien financier de l'association France-Parkinson, de l'AP-HP (PHRC n AOM 95006), de la Communauté Européenne (contrat Biomed $2 \mathrm{n}^{\circ}$ BMH4CT 96066) et de la Deutsche Forschungsgemeinschaft.

\section{Alexandra Dürr \\ Christoph Lücking \\ Alexis Brice \\ pour le Groupe français \\ de recherche génétique \\ sur la maladie de Parkinson (PDG)}

Inserm U. 289, Hôpital de la Salpêtrière, 47, boulevard de l'Hôpital, 75651 Paris Cedex 13, France.

\section{TIRÉS À PART}

A. Brice. 